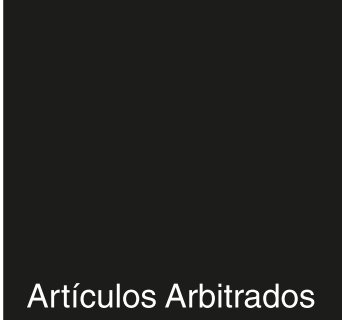

\title{
Emocionalidad y espacio público: detenciones arbitrarias de jóvenes de sectores populares de Córdoba (Argentina)
}

\section{Andrea Bonvillani}

Doctora en Psicología, Universidad Nacional de Córdoba (UNC). Posdoctorado del Centro de Estudios Avanzados UNC. Exbecaria de doctorado y posdoctorado CONICET. Profesora a cargo en Teoría y técnicas de grupo y profesora asistente en Psicología social, Facultad de Psicología, UNC. Directora de distintos proyectos de investigación a nivel local e internacional en el cruce de temáticas juventudes-política-subjetividades. Integrante del grupo de trabajo del Consejo Latinoamericano de Ciencias Sociales (CLACSO) "Juventudes, Infancias: Instituciones Sociales, Políticas y Culturas en América Latina”. Excoordinadora del grupo de trabajo CLACSO "Ciudadanías críticas, subjetivaciones y transformaciones sociales”, período 2013-2016. Pertenencia institucional CiPsi/ CIECS-CONICET, Facultad de Psicología, UNC.

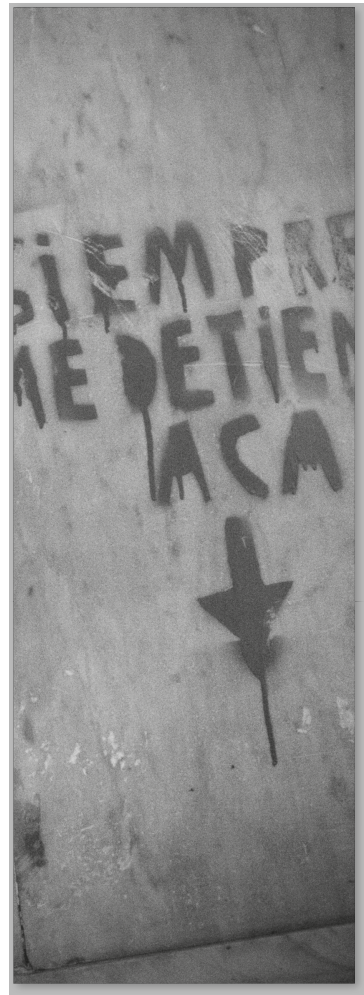




\title{
Resumen
}

Este trabajo es el resultado de una investigación en curso, y se propone analizar las experiencias de uso del espacio público de jóvenes de sectores populares de Córdoba (Argentina). Estas están atravesadas por las denominadas "detenciones arbitrarias": prácticas que se desarrollan de manera cotidiana y mayoritariamente en la calle cuando los jóvenes son demorados y violentados por los agentes policiales, sin mediar la comisión de un delito, sino simplemente la sospecha de que podrían realizarlo. El artículo focaliza en la dimensión subjetiva, particularmente en el registro emocional de los jóvenes que las padecen, y tiene como horizonte una reflexión en torno a la accesibilidad (real) a determinados espacios que son (formalmente) "públicos". Se desarrolló una investigación cualitativa que permitió concluir que la reiteración cotidiana de la experiencia de ser detenido y violentado produce una emocionalidad de indefensión e impotencia. En el espacio urbano cordobés se desarrolla esta escena cotidiana, donde los sujetos juveniles parecen ser meramente cuerpos que son sustraídos de la calle para ser trasladados y depositados en otro lugar. Se trata de una operatoria policial compulsiva que actualiza de manera constante una paradoja: la calle es pública, para algunos.

\section{Palabras clave}

Jóvenes; espacio público; emocionalidad; Córdoba.

\begin{abstract}
Public space and youths' emotional behaviour: arbitrary detention of young people of popular sectors in Córdoba (Argentina). This work is the result of ongoing research and attempts to analyze the experiences of young people from popular sectors of Córdoba (Argentina) in their use of public space. Those experiences are traversed by "arbitrary arrests": practices that are carried out every day, mostly on the street when young people are delayed and harassed by police agents without having committed a crime, based only on the suspicion that they could do so. This paper focuses on the subjective dimension, particularly regarding the emotional register of the young people who suffer these detentions leading to a horizon a reflection on the effective accessibility to certain spaces that are formally "public".

A qualitative investigation was developed that concluded that the daily reiteration of the experience of being stopped and harassed produces emotions of helplessness and impotence. Urban space in Cordoba is the scene where these daily events develop, where juvenile subjects seem to be merely bodies that are taken from the street to be transferred and deposited elsewhere. It is a compulsory police operation that constantly updates a paradox: the street is public, only for some.
\end{abstract}

\section{Keywords}

Young people; public space; emotions; Córdoba. 


\section{Introducción}

Este trabajo es el resultado de una investigación que se desarrolla hace algunos años ${ }^{1}$, y se propone analizar las experiencias de uso del espacio público de jóvenes de sectores populares de Córdoba (Argentina). Estas están atravesadas por las denominadas "detenciones arbitrarias": prácticas que se desarrollan de manera cotidiana y mayoritariamente en la calle, cuando los jóvenes son demorados y violentados por los agentes policiales, sin mediar la comisión de un delito, sino simplemente la sospecha de que podrían realizarlo. Este artículo focaliza en la dimensión subjetiva, particularmente en el registro emocional de los jóvenes que las padecen, y tiene como horizonte una reflexión en torno a la accesibilidad (real) a determinados espacios que son (formalmente) "públicos”.

Se parte del supuesto de que el espacio urbano está sometido a un régimen de uso (CASTELLS, 1984) que es, a su vez, la plasmación de la estratificación social. En consecuencia, se trata de un ejercicio de soberanía por parte de los grupos sociales que detentan poder (DuHAU, 2003).

La relación conflictiva entre el espacio urbano y el ejercicio de la ciudadanía fue intuida hace varias décadas (LEFEBVRE, 1972), al identificar formas de segregación planificada que excluyen a determinados grupos sociales del goce de los bienes de la ciudad. Así, la geometría urbana se constituye en un complejo sistema de barreras y habilitaciones y configura la experiencia cotidiana de la desigualdad social: vivir día tras día la dificultad de acceder a determinados puntos de la trama urbana y, simultáneamente, la confinación en otros.

En este marco, el espacio urbano es simultáneamente escenario y objeto de conflictos, en los que se disputa fundamentalmente su carácter "público”, al ser este un concepto polisémico (Delgado y Malet, 2008), ya que remite de manera simultánea a lo que es de todos, lo común, pero también a lo que no tiene restricciones en su acceso, lo cual lo transforma entonces en el locus privilegiado para el encuentro social que permite la coincidencia entre "extraños", el desarrollo de un tipo de sociabilidad propia de las urbes. Otra vertiente enfatiza en su ligazón con el Estado: el espacio público se define, entonces, como lo no privado.

Finalmente, en un sentido político, el espacio público se entiende como esfera deliberativa, en la cual es posible el acuerdo entre personas autónomas y libres, que plasma el ideal burgués de democracia, que parte de la libertad e igualdad de todos frente a la ley. Se trata, por

\section{Focaliza en la} denominada "Marcha de la gorra", acción colectiva que agencian grupos de jóvenes cordobeses desde hace una década para demandar el cese de políticas de seguridad del gobierno provincial que los hostigan, maltratan y eventualmente los matan. Un eje de esta indagación son las situaciones cotidianas de violencia policial que los jóvenes sufren como parte de esta política de Estado, $y$ que se desarrollan en el espacio público regulando su uso. En el bienio 2014-2015, el estudio se denominó "La 'Marcha de la gorra' como experiencia de subjetivación política de jóvenes de Córdoba (Argentina)", y desde el 2016 a la actualidad, "Diez años de la Marcha de la gorra: memorias de una lucha juvenil cordobesa". En ambos casos, con la dirección de la autora y con un subsidio de la Secretaría de Ciencia y Técnica de la Universidad Nacional de Córdoba. Lugar de trabajo: Facultad de Psicología de la misma universidad. 
consiguiente, de la "concreción física en que se dramatiza la ilusión ciudadanista" (Delgado y MALET, 2008: 59), aquella por la cual no existen contradicciones ni conflictos porque son superados por el supuesto acceso y participación igualitaria. Justamente porque se trata de una abstracción formal y de una idealización, es necesario desnaturalizar el sentido público del espacio social, propósito que guía este trabajo.

El espacio urbano no es meramente un territorio físico, sino una construcción psicosocial: "lo urbano es actuado, modelado, disputado, apropiado e inscrito en los cuerpos de sus habitantes” (Lindón, 2015: 9). A partir de este supuesto, el artículo se interroga por el registro de percepciones y emocionalidad que la experiencia del espacio urbano (público) produce en los jóvenes de sectores populares de Córdoba, a partir de constituirse en un locus privilegiado de conflictividad con la policía, como se explica a continuación.

La problemática del miedo asociado al delito en las grandes ciudades se instala desde hace varias décadas (ENTEL, 2007), genera una fuerte demanda por mayor seguridad "en las agendas periodísticas, políticas y electorales y se convierte en un eje central de difusión de una comunicación política asociada a la violencia urbana en América Latina en general, y Argentina en particular" (CALZADO y GALLARDO, 2016: 3). Este proceso ha sido inscripto en la modernidad tardía y tiene como motor el temor a perder los bienes materiales acumulados, que alimenta un clima de amenaza permanente, frente al cual las sociedades responden construyendo barreras materiales y simbólicas e intensificando todos los controles posibles, a la vez que refuerzan los prejuicios sobre los “otros”, considerados esencialmente peligrosos (YounG, 2007). La "otrificación” consiste en la atribución a un "otro” de rasgos negativos de manera esencialista. Esta demonización permite consolidar un "nosotros" que se autopercibe como "la gente buena” y se construye en oposición a "los malos”, los "peligrosos", a los que se les atribuye ser la causa de todos los riesgos sociales.

En Latinoamérica los jóvenes pobres son generalmente objetos de otrificación. Como afirman varias autoras (Regulllo, 2003; ChAVEs, 2005), en nuestras sociedades se refuerza un imaginario que les imputa la condición de enemigo interno, responsable inequívoco de la violencia y la inseguridad urbana y, consecuentemente, objeto de represión. El que se los considere de esta forma justifica prácticas punitivas que van desde controles permanentes en la vía pública hasta desapariciones y muertes. Desde la criminología crítica se sostiene 


\section{Emocionalidad y espacio público:}

un enlace entre estos imaginarios sociales y el accionar de las fuerzas policiales, las cuales ejercen una forma de control social sobre los jóvenes pobres mediante la humillación, el castigo y la sumisión, con un proceso de etiquetamiento vinculado con su condición de clase, y que termina constituyendo una "intolerancia selectiva" (WACQUANT, 2000).

En Argentina las políticas estatales ejecutadas por estas fuerzas configuran una rutina cotidiana marcada por la persecución y la violencia física y simbólica respecto de estos jóvenes. Como lo afirma un estudio reciente (CELS, 2016), aunque la libre circulación es un derecho consagrado por la Constitución Nacional, las prácticas de control y detención realizadas de manera constante por las fuerzas de seguridad afectan de modo particular a estos grupos.

En una investigación realizada en Córdoba (BolatTI ET ÁL., 2012), se constata que el $78 \%$ de los jóvenes encuestados fueron detenidos en la vía pública por la policía sin una razón aparente. Cuando se trata de jóvenes de barrios pobres, esa proporción alcanza el 95,8 \%, lo que indica que la accesibilidad al espacio público cordobés resulta limitada para ellos.

De acuerdo con las escasas y desactualizadas cifras con las que se cuenta por parte de los órganos oficiales de la provincia de Córdoba, entre 2005 y 2011, las detenciones arbitrarias crecieron en más de un $700 \%$; de estas, un $70 \%$ afectaron a jóvenes, fundamentalmente, varones y pobres (BRocCA ET ÁL., 2014).

En síntesis, el acceso y disfrute del espacio público de los jóvenes de sectores populares de Córdoba están condicionados por un accionar represivo de la policía en la calle ${ }^{2}$, que a su vez remite a la política de seguridad del gobierno provincial, la cual fue caracterizada por diversos autores (Brocca et Ál., 2015; Plaza Schafer y Morales, 2013) como violenta, discrecional y discriminatoria.

Uno de los lineamientos centrales del esquema de seguridad provincial es la prevención, lo que implica permanentes controles y pedidos de identificación en la vía pública. Estas intervenciones están inspiradas en la llamada Doctrina de la tolerancia cero, que busca anticiparse a la comisión del delito a partir de la focalización de la vigilancia sobre los que se supone son productores de inseguridad: básicamente sujetos pobres o que tienen comportamientos incivilizados, de acuerdo con prejuicios sociales, raciales y culturales (WACQUANT, 2000).
2. A este esquema debemos sumar las denominadas "políticas de ordenamiento territorial" del gobierno cordobés, que consisten en la creación de "barrios ciudad", donde se relocaliza a pobladores de asentamientos irregulares desde el centro a la periferia urbana. La expulsión y segregación de estos grupos sociales, así como su fijación en puntos determinados, configuran un régimen de uso del espacio urbano que no solo permite el control de los sindicados como peligrosos, sino la realización de importantes negocios inmobiliarios a partir del usufructo de los predios céntricos de donde se los desaloja. Para ampliar véase BoIto y MicheLAZzo (2014). 
3. Se trata del "Colectivo investigador Marcha de la gorra", integrado por investigadoras con distintas trayectorias y procedencias disciplinares. Se realiza un trabajo cooperativo en todos los momentos correspondientes a un estudio cualitativo, por lo cual los fragmentos discursivos que se utilizarán como respaldo empírico para la construcción de las categorías son producidos y compartidos solidariamente.

4. Hablar de "jóvenes" resulta problemático: puede sugerir adhesión a un mero criterio de edad biológica. Por el contrario, en este contexto se la utiliza connotando la diversidad de modos de vivir la juventud, de acuerdo con pertenencias sociales, culturales, étnicas y de género. En este marco, el uso del masculino "jóvenes"

busca facilitar la lectura. No debe interpretarse como un sesgo machista.

5. Véase cita número 1.
Son innumerables las medidas que verifican la orientación represiva de la seguridad en Córdoba, entre las que destaca un incremento desmesurado del presupuesto en este rubro (744 \% en siete años), que se particulariza en el crecimiento exponencial del personal de la fuerza (107\% desde 2005 a la actualidad), lo cual hace que la ratio policías/ciudadanos cordobeses esté muy por encima de las recomendaciones de la Organización de las Naciones Unidas (Jов у NAHAs, 2016).

Estos apuntes introductorios buscan contextualizar el análisis sobre las experiencias de los jóvenes populares de Córdoba — el cual se emprende en la tercera sección de este artículo- cuando transitan y viven el espacio público local, mientras que a continuación se explicita el encuadre metodológico de la investigación que lo originó.

\section{Metodología}

Se desarrolló una estrategia de investigación cualitativa, en tanto se buscó explorar los sentidos subjetivos que los jóvenes construyen en su vida cotidiana en la ciudad. Se despliega un conjunto de técnicas de producción de datos, que ponen en primer plano la relación social entre las investigadoras ${ }^{3}$ y los jóvenes ${ }^{4}$. En ese marco, las situaciones de persecución y abuso policial constituyen una dimensión para ser reconstruida en las distintas instancias de acceso al campo de estudio, de las cuales se seleccionan para este artículo las siguientes:

- "Conversaciones en marcha”, es decir, diálogos informales con aquellos que participan de la manifestación, objeto del estudio a partir del cual se realiza este artículo $^{5}$. Los marchantes son abordados en el mismo momento en que realizan la acción, intentando con ellos pensar sobre esta y "acompasar la tentativa reflexiva con el caminar, saltar, gritar, cantar, bailar, etc.” (AuтоRA, 2016: 1604). Por lo tanto, son más breves y focalizadas que las otras técnicas empleadas.

- Entrevistas en profundidad (VALLÉs, 1999), realizadas a los jóvenes que se articulan a la marcha a través de distintos espacios (agrupaciones estudiantiles, organizaciones barriales, colectivos artísticos y culturales, organizaciones no gubernamentales, etc.). A diferencia de la herramienta anterior, se trata de interacciones verbales pautadas de antemano con el entrevistado, en un espacio-tiempo 
distinto del de la marcha. De acuerdo con los criterios propios de la metodología cualitativa, el número de entrevistas que realizar no puede determinarse de antemano, sino que resulta de la "saturación", es decir, la emergencia de redundancia en las respuestas obtenidas ${ }^{6}$.

- Talleres grupales de reconstrucción de experiencias, intercambio y construcción colectiva (AuTORA, 2011), que se realizaron en establecimientos educativos de nivel medio de la ciudad capital y otras localidades de la provincia de Córdoba ${ }^{7}$, durante 2016. Inspirados en las concepciones socio-educativas de la Educación Popular, en los talleres se utilizaron distintos recursos grupales y lúdicos, para favorecer la expresión y la comunicación de los jóvenes sobre sus distintas experiencias en torno a la policía. En la práctica esta técnica opera como sucesivas entrevistas grupales, donde se potencia la interacción entre los jóvenes y con un investigador que coordina los talleres, propone disparadores para la discusión y favorece la producción de preguntas y el diálogo entre los participantes. Los registros textuales son producidos por un investigador-observador que acompaña cada una de las actividades.

El análisis se orientó a la construcción de ejes y categorías a partir de las recurrencias y divergencias encontradas en el trabajo de campo. Se utilizó el paquete informático Atlas.ti.

Resultados y discusión

"Parar", "levantar" y "llevar"

De acuerdo con una investigación realizada en distintas ciudades de la Argentina, "las interceptaciones policiales, las demoras y las detenciones sin orden judicial en las que no hay una situación de delito flagrante" (CELS, 2016: 20) ${ }^{8}$ se constituyen en situaciones cotidianas para los jóvenes de sectores populares, como se constata en el estudio que origina este artículo. No se trata de episodios aislados; por el contrario, para muchos jóvenes son vivencias reiteradas a lo largo del tiempo, que terminan formando parte de su vida diaria. El registro discursivo muestra una presencia policial permanente y amenazante, que actúa cortando el paso, obstaculizando la circulación de algunos jóvenes o —como se precisa más
6. Atendiendo al "muestreo teórico" propuesto por la teoría fundamentada en los hechos, se tuvieron en cuenta como criterios el género, la pertenencia organizativa, la exposición a experiencias de detención policial y la trayectoria de participación en la acción de protesta en estudio. El rango de edad de los sujetos va de los dieciséis a los veintiocho años, $y$ asumiendo - como ya se precisó-que las experiencias de detención en la vía pública alcanzan particularmente a jóvenes de sectores populares, este trabajo focaliza en sus testimonios y en los de algunos adultos que acompañan su socialización. Se tomaron en total veintitrés entrevistas en profundidad.

7. En este artículo se analizan los registros de los cinco talleres llevados a cabo en un Instituto Provincial de Educación Media (en adelante, IPEM) de la ciudad de Colonia Caroya, cercana a la capital cordobesa, en el marco del proyecto de investigación antes mencionado.

8. El abuso policial en Córdoba se despliega además en prácticas de "allanamientos violentos" (BROCCA ET ÁL. 2015) que violan el espacio privado de la casa, a través de operativos de "intervención territorial que implican la presencia masiva de efectivos en barrios pobres por períodos de tiempo variables" (CELS, 2016: 13). 
9. Los fragmentos textuales se citan entrecomillados y se consigna nombre ficticio del hablante para preservar su anonimato, así como su pertenencia organizativa.

Finalmente se indica la técnica de la cual resulta dicho fragmento y su año de realización.

10. El abuso policial presenta matices de acuerdo con el género.

Las jóvenes son víctimas de miradas o palabras que incomodan y que

tienen un claro contenido sexual: "me pasa más seguido, cuando salimos a bailar, que el policía, por ejemplo, nos dice cosas...

y yo nunca le contesto,

o se ponen agresivos, ya

de pasar de piropos a insultarnos" (Rita, grupo juvenil de una ONG, entrevista en profundidad, 2015). Justamente porque forman parte de un sistema de violencia simbólica machista, estas prácticas policiales tienden a no ser reconocidas como tales por las propias víctimas, $y$ suelen invisibilizarse como ejercicios de seducción. Es una dimensión para ser profundizada en futuros estudios. adelante- impidiendo que ocupen determinados puntos de la geografía urbana. En algunos casos, en que se puede establecer una recurrencia respecto del agente concreto que realiza la práctica, es posible hablar de acoso policial:

"A mí en un lapso de dos meses me pararon ocho veces, una vez por semana. El hostigamiento todos los días, que te va pasando" (Luis, organización juvenil barrial, entrevista en profundidad, 2015). ${ }^{9}$

"Me pararon la semana pasada, dos veces volviendo a mi casa, pero tranquilo, me pidieron documento" (Leandro, Tercer taller IPEM, Colonia Caroya, 2016).

"A mí me paran todos los días, siempre el mismo" (Walter, Cuarto taller IPEM, Colonia Caroya, 2016).

"Pero que me han parado, se cansaron" (Omar, Segundo taller IPEM ,Colonia Caroya, 2016). "Cada vez que hacíamos un taller en el grupo de jóvenes, teníamos una cantidad de grupos sin gente porque los detenía la policía, era como una cosa que todo el tiempo así pasaba” (Marta, técnica de grupo juvenil ONG, entrevista en profundidad, 2015).

"Tal vez a mí no me paran, pero tengo seis hermanos varones ${ }^{10}$, que lo vivo constantemente de ir a buscarlos porque los alzan, porque los llevan constantemente por contravenciones" (Gilda, familiar de víctima de gatillo fácil. Conversación en marcha, 2014).

La reiteración cotidiana de la experiencia de ser detenido y violentado produce una emocionalidad de indefensión e impotencia, porque el hostigamiento policial es significado por los jóvenes como sin salida:

"No hay forma de no volverlos a cruzar. Acá no hay forma de esquivarlos" (Leandro, Cuarto Taller IPEM, Colonia Caroya, 2016).

“Acá te los seguís cruzando y no te dejan tranquilo. Si los denuncias te pegan...” (Héctor, Primer Taller IPEM, Colonia Caroya, 2016).

La producción de un sentido sobre la experiencia vivida se expresa en una especie de jerga que permite designar las distintas instancias o modalidades del proceso. "Parar", "levantar" y "llevar" son acciones indicativas de la situación de pasividad en la que viven estas experiencias con la policía: los jóvenes parecen ser objetos de la práctica de otros. El espacio público es el escenario donde se desarrolla esta escena cotidiana, donde los sujetos juveniles parecen ser meramente cuerpos que son sustraídos de la calle para ser trasladados y depositados en otro lugar. Se trata de una operatoria policial compulsiva que actualiza de manera constante una paradoja: la calle es pública, para algunos. 
Emocionalidad y espacio público: detenciones arbitrarias de jóvenes de sectores populares de Córdoba [Argentina]

"Me llevaron, me sacaron todo y me metieron a la celda" (León, Segundo taller IPEM Colonia Caroya, 2016).

"A unos chicos los llevaron, estaban haciendo malabares en la plaza de noche y pasó la policía y los llevó, por estar haciendo malabares" (Karen, murga ${ }^{11}$, entrevista en profundidad, 2015). "No nos dijeron porque me levantaron (...) y eso está mal, yo tenía trece años" (Omar, Segundo taller IPEM, Colonia Caroya, 2016).

Los relatos de las víctimas muestran en detalle las distintas formas que puede asumir la violencia cotidiana de la policía en las calles de la ciudad. Estas modalidades pueden ser interpretadas en un sentido procesual, que va desde el ser parado para un control, hasta ser demorado y llevado a la comisaría. La proyección de estos momentos supone un gradiente de violencia policial que va desde el plano simbólico al físico, pudiendo causar incluso la muerte $^{12}$. El desarrollo de este proceso es contingente. Como sostiene uno de los entrevistados: "soy del grupo de los que detienen y no se llevan. Pero hay muchos que por ahí capaz, el policía ya lo vio una vez y la próxima vez que se los cruzó los pararon y se los llevaron, por 'merodeo' (entre comillas), pero la bronca venía de otro lado. O a veces, no sé, porque tienen que llenar números y se los llevan porque ya se los llevaron alguna vez y bueno, ya está, si ya nos lo llevamos, lo llevamos de vuelta, y cosas así. Después sí, está el grupo de los que no paran nunca, que bueno me parece que también es más la gente que da otra impresión a lo mejor, con esto de los prejuicios que veníamos hablando, que andan o todo el tiempo bien vestida, así, o entre comillas” (Esteban, grupo juvenil de una ONG, entrevista en profundidad, 2015).

La categorización espontánea que realiza el joven pone en evidencia la arbitrariedad con la que se manejan los agentes policiales en la vía pública. De acuerdo con este y muchos otros relatos, el motivo por el que se los detiene no es la comisión de un delito, sino la sospecha de la potencialidad de producirlo. Esta discrecionalidad se manifiesta tanto en una primera selección que determina quién será "parado" en la vía pública como en un segundo momento, cuando se elige quién será "levantado" y "llevado" a una sede policial. Los relatos muestran un manejo prejuicioso por el cual el policía produce un marcaje selectivo que determina quién será retenido en el espacio público y quién podrá transitar sin inconvenientes.

El núcleo de este prejuicio policial parece alojarse en la apariencia de algunos jóvenes, es decir, lo que muestran, y puede verse a nivel de la superficie de sus cuerpos. En lenguaje local "la pinta” refiere a la impresión que el otro deja en mí a partir de su aspecto: cómo se
11. Forma de expresión artística que combina música popular con teatralidad, utilizando instrumentos de percusión, coros y movimientos corporales altamente ritmicos. Se desarrolla especialmente en Argentina y Uruguay.

12. En 2015 en la provincia de Córdoba se contabilizaron quince jóvenes muertos por responsabilidad de las fuerzas policiales (BROCCA ET ÁL., 2015). 


\section{Andrea Bonvillani}

13. Categoría local, "ladrón". viste, cómo se peina. Este registro permite entonces ubicarlo en unas categorías previamente incorporadas desde las cuales se da sentido a esa forma de presentación:

"Que no puede haber tanta discriminación y tanto prejuicio por una persona que lleva una gorra, un arito en la cara. La portación de rostro, que si vos salís a la esquina con una gorra de noche o tenés un arito en la cara o tenés pinta de negro villero, te levantan, te llevan, te pintan los dedos" (Karen, murga, entrevista en profundidad, 2015).

"Depende del sector social, porque la juventud en general no... pero también el poder económico, la forma de vestir, todo lo que y a sabemos. Claro, si te ven con ropa deportiva. O por el color de piel también, porque hay chicos que se visten no con ropa deportiva, pero por el color de piel los paran, no tienen documentos, se los llevan. Y piercing, cortes de pelo así como el mío [se ríe], ropa de gimnasia” (Benjamín, manifestante autoconvocado, entrevista en profundidad, 2015). "Es injusticia porque agarran al mal vestido porque es 'el choro"13" (Blas, Primer Taller IPEM Colonia Caroya, 2016)

Como muestran los testimonios de los jóvenes que han sido víctimas de este proceso y las abundantes referencias en la literatura (NuÑEz, 2006; AutorA, 2015), existe una asociación casi automática entre las opciones estéticas que portan los cuerpos de los jóvenes de sectores populares y la atribución de peligrosidad vinculada con la producción del delito urbano. "Profiling" es el concepto con el que se designa una atribución causal al comportamiento de las fuerzas policiales, ligado a sesgos étnicos y de clase, que constituye una práctica discriminatoria (ZAVALETA ET ÁL., 2016).

En la ciudad de México, según una investigación reciente, es habitual que la práctica de detener y eventualmente revisar a personas que transitan en la vía pública sea conducida por fuertes prejuicios de los policías: "Definir un comportamiento como merodear o 'actitud sospechosa' se entreteje con estereotipos sociales sobre la edad, el sexo y la apariencia: por ejemplo, joven, hombre y 'mal vestido'” (Alvarado y Silva, 2013: 459).

Un punto para subrayar es que el estigma que porta el joven en su cuerpo es una marca cultural que denota su procedencia social, porque su apariencia desacreditada y sospechada remite al desvío respecto de una estética hegemónica; por eso se habla de "mal vestido". Lo que se rechaza del aspecto y se identifica de manera naturalizada con el delito no es solo una adhesión estética: aparecen referencias al fenotipo en el color de piel, de ojos y de pelo. El componente racial está presente en la forma de estigmatización nativa a través de la 
Emocionalidad y espacio público: detenciones arbitrarias de jóvenes de sectores populares de Córdoba [Argentina]

figura del "negro villero", que remite a la "racialización de las relaciones de clase" (MARGULIs, 1999: 38), ya que en la Argentina en general no abundan personas de piel negra, por lo que esta apelación para denostar al otro es una atribución de procedencia social y estilo de vida ("villero"). En conclusión, la arbitrariedad policial para detener en la vía pública se basa en un a priori de selectividad, en-carnado en los jóvenes populares:

"No, a todos no afecta por igual... Porque es una cuestión que, más vale que al rubio no le afecta igual que al morocho o que le falta un diente o que usa gorrita" (Lautaro, murga. Conversación en marcha, 2014).

"Si vos vas y ves a un chico rubio de ojos claros, no lo paran, y va uno de los chicos morocho con gorra, te lo paran y te lo llevan, y hoy en día no te lo llevan solo, te lo golpean, los torturan” (Gilda, familiar de víctima de gatillo fácil. Conversación en marcha, 2014).

"No se meten con los de plata o vestidos bien. Piensan que sos chorro, que te drogás y nada que ver, ite estigmatizan!” (León, Cuarto Taller IPEM, Colonia Caroya, 2016).

Para estos jóvenes la experiencia de la calle en Córdoba produce sentimientos de injusticia, de discriminación, que derivan de una recurrente práctica de juzgamiento y sospecha.

\section{Entrar y salir.}

\section{Inaccesibilidad a determinados espacios y fijación de los cuerpos en otros}

El grafiti de la página 118 se encuentra sobre una de las paredes del Cabildo histórico de la ciudad de Córdoba, en su corazón. Es una marca en el cuerpo de la ciudad que hace presente una práctica que parece ser invisible a los ojos de todos, aunque sea constante y a plena luz del día.

El análisis de los relatos de los jóvenes permite trazar una suerte de topología de las detenciones en la trama urbana cordobesa. Sería posible reconstruir una cartografía de la persecución y el maltrato policial, que uniera zonas y puntos que a fuerza de prácticas de barrido policial resultan prohibidos para los jóvenes pobres de Córdoba:

"Está simbolizando en medio de la plaza San Martín", que ahí sí te paran, y te trae como esta cuestión, te está trayendo a la memoria siempre que esto está pasando, y que se muestre, que se vea, que la gente lo vea. Que la gente que no lo piensa, que la gente que le chupa un huevo esto, vea. Acá está pasando, todo el tiempo están deteniendo" (Luis, organización juvenil barrial, entrevista en profundidad, 2015).
14. Es la plaza mayor de la ciudad, ubicada en pleno casco histórico y en el actual microcentro. 


\section{Andrea Bonvillani}

15. Uno de los centros comerciales más importantes de la ciudad, ubicado en su zona céntrica.

16. El espacio verde más grande de la ciudad. $\mathrm{Ha}$ sido el punto de encuentro

y esparcimiento para varias generaciones de cordobeses.

17. Categoría local, "policía".

Grafiti. Autor: Colectivo Manifiesto

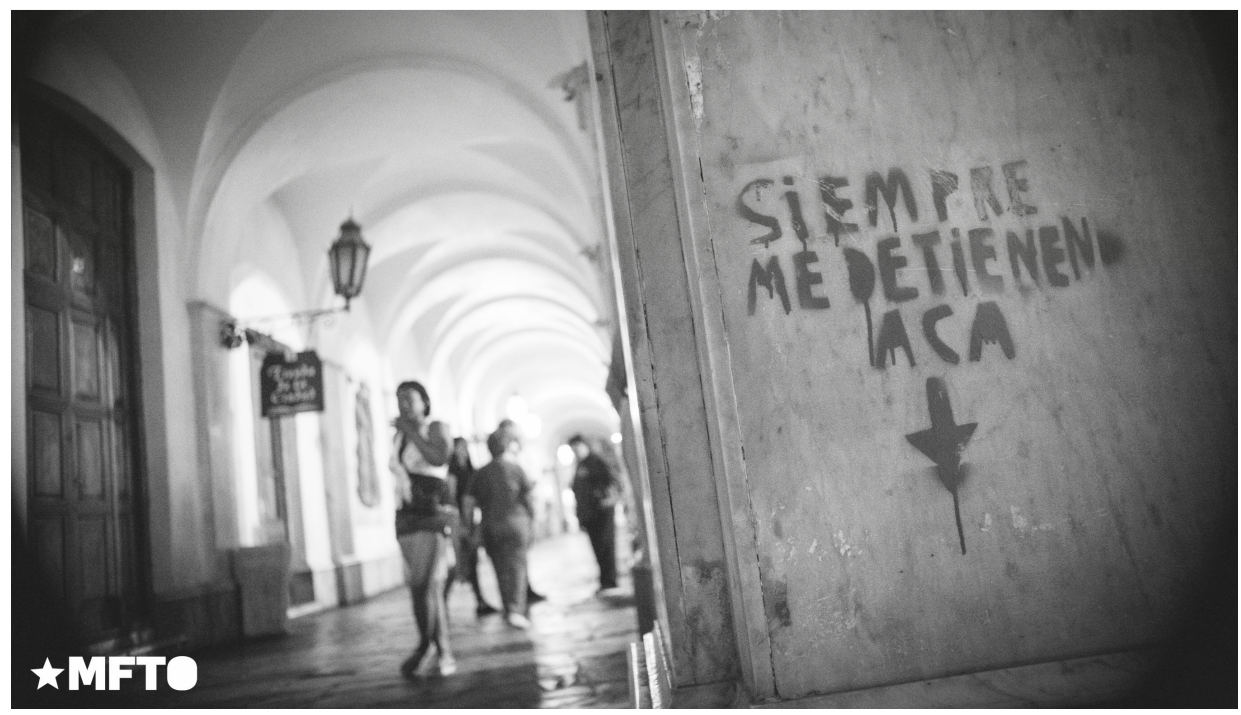

"Una vez, estaba con un amigo sentado en la puerta del Patio Olmos ${ }^{15}$, esperando a otro amigo más, y vino la policía y nos pidió los documentos. Nosotros estábamos sentados como tanta gente que había ahí, y bueno. Yo estaba vestido como me visto, él estaba vestido como se viste, así con gorra, que sé yo, y nos piden los documentos” (Esteban, grupo juvenil de una ONG, entrevista en profundidad, 2015).

"Era muy común que había un montón de chicos que faltaban el lunes porque el domingo habían ido al Parque (Sarmiento) ${ }^{16}$, y bueno, entraba en la posibilidad de caer en cana ${ }^{17}$ por estar en el parque, paseando con la novia, con el novio..." (Marta, técnica de grupo juvenil ONG, entrevista en profundidad, 2015).

"Cuando los chicos, por ejemplo, yo, salgo del colegio y me quiero dirigir al Patio Olmos, un sector bastante turístico, los policías te ponen como barreras, como que esconden la basura debajo de la alfombra, por decirlo de una manera” (Benjamín, manifestante autoconvocado, entrevista en profundidad, 2015).

"Me acuerdo una vez que iba en el colectivo, me encuentro con un grupo, con estos chicos, dos de quince años y los reconozco. Se iban al parque, y me pidieron que los acompañara 
Emocionalidad y espacio público: detenciones arbitrarias de jóvenes de sectores populares de Córdoba [Argentina]

y me bajara con ellos, porque en realidad iban con una sensación de mucha preocupación de que no pudieran llegar (...) Yo les dije que yo me bajaba en el Buen Pastor ${ }^{18}$ y me preguntaron ‘¿nos podemos bajar con vos?’, y ahí me dijeron que nunca habían podido pasar por el Buen Pastor porque era obvio que los detenían. Entonces, fueron conmigo, hicieron el recorrido conmigo y después se fueron al parque" (Marta, técnica de grupo juvenil ONG, entrevista en profundidad, 2015).

A través de estas prácticas reiteradas todos los días, la policía se constituye en garante de la arbitrariedad, ya que no existe un motivo efectivo por el cual los jóvenes debieran ser retirados de estos espacios. Se va conformando así un régimen de uso del espacio de la ciudad, donde existen barreras efectivas: los jóvenes de sectores populares evitan llegar y permanecer en puntos específicos de concentración de poderes económicos (como centros comerciales y parques) o capitales simbólicos (como el casco histórico).

La experiencia de lo urbano se inscribe en el cuerpo de los jóvenes: el miedo a ser detenido y maltratado se vuelve paranoia y autocensura confirmando así a nivel subjetivo la exclusión social y urbana, y por ello, haciéndola más eficaz. Algunos, incluso, optan por no ir al centro, para evitarse un nuevo padecimiento del que se tiene certeza:

"Yo me he ido de la ciudad y ahí he notado como la paranoia que tengo todo el tiempo, ves un móvil y, jta! De ponerme alerta” (Lautaro, murga. Conversación en marcha, 2014).

"L a policía está para proteger y servir a los ciudadanos y nosotros no estamos protegidos, nos sentimos perseguidos” (León, Primer Taller IPEM, Colonia Caroya, 2016).

"Mario no iba al centro, porque se bajaba del colectivo y ya tenía veinte policías ahí esperándolo; entonces directamente no iba al centro" (Rita, grupo juvenil de una ONG, entrevista en profundidad, 2015).

En un informe reciente, se identifican los "desalojos de los espacios públicos de encuentro y recreación” (BROCCA ET ÁL., 2015: 22) como una de las modalidades más frecuentes de ejercicio de abuso policial sobre los jóvenes cordobeses, situación que ilustra la experiencia cotidiana de aquellos que practican murga en las plazas y parques:

"A mí me toca muy mucho (...) por el tema de la policía, de querer venir a sacarte de los espacios públicos. $O$ sea, este espacio es donde ensayamos nosotros, y que te venga a querer sacar la policía es como que te vengan a querer sacar de tu casa" (Mariano, murga, entrevista en profundidad, 2013).
18. Complejo cultural ubicado en el corazón del barrio Nueva Córdoba, uno de los de mayor poder adquisitivo. 


\section{Andrea Bonvillani}

"Tenemos esa dificultad para hacer arte digamos libremente, en una calle o un espacio" (Carlos, murga, entrevista en profundidad, 2013).

'Se los llevan por ser 'hippie mugroso', como los chicos de la murga y a los que andan en la plaza haciendo nada” (Karen, murga, entrevista en profundidad, 2015).

La murga es una práctica artística que supone la producción de sonidos estridentes, bullicio, movimientos corporales dislocados, y que convoca a un gran grupo de personas para hacer un uso del espacio público que, desde las pautas hegemónicas, podría considerarse inapropiado. Estas características la hacen un blanco privilegiado de la operatoria policial enmarcada en la doctrina de la tolerancia cero, ya que su propósito preventivo del delito impone un control represivo de los comportamientos incivilizados. Prejuicio mediante, es posible que esto "mal visto" sea reprimido por "incívico, o sea contraventor de los principios abstractos de la 'buena convivencia ciudadana”" (Delgado y Malet, 2008: 60).

En algunos entrevistados comienza a esbozarse a nivel discursivo el sentido del espacio público como un derecho que va a ser disputado, mostrando que lejos de ser un objeto armónico, es territorio de lucha por su ocupación y goce:

"No estamos molestando, estamos luchando por nuestros derechos como ciudadanos y como murgueros a la hora de ocupar un espacio público; y por los derechos de los demás” (Carlos, murga, entrevista en profundidad, 2013).

"Nosotros particularmente ensayamos en plazas y en espacios abiertos y notamos como últimamente está más complicado poder apropiarse de un espacio público, e ir simplemente a cantar a un espacio así ya está mal visto, o a tocar la guitarra ya es motivo para que la policía te caiga y tenga pleno derecho a molestarte (...) nosotros más allá de lo que individualmente vivimos, del acoso y esa cosa constante, nos molesta mucho no poder utilizar un espacio público para ensayar" (Lautaro, murga, conversación en marcha, 2014).

Esta restricción en el uso del espacio que figura en los papeles como accesible a todos tiene varias consecuencias prácticas. En primer término, implica una privación de las posibilidades de inserción social, laboral, educativa:

"Y pierden el trabajo" (Gilda, familiar de víctima de gatillo fácil. Conversación en marcha, 2014). "Acá a un baile no puedo ir, me tengo que desnudar antes de entrar... te ven la cara, no podes salir de noche” (Héctor, Primer Taller IPEM, Colonia Caroya, 2016).

"No hay muchos lugares para que ellos salgan. Los hacen desvestir; no van a los bailes ya" 
Emocionalidad y espacio público: detenciones arbitrarias de jóvenes de sectores populares de Córdoba [Argentina]

(Bárbara, Profesora de Ciencias Naturales, Primer Taller IPEM, Colonia Caroya, 2016). "Muchos chicos no llegaban a educación física porque quedaban en el camino. Los paraba la policía, no tenían documentos, se los llevaban” (Benjamín, manifestante autoconvocado, entrevista en profundidad, 2015).

Por otra parte, produce una exclusión social territorial que obliga a los jóvenes pobres a quedarse en los lugares donde residen, fijando sus cuerpos en un sitio controlable:

" $Y$ los chicos evitan de salir del barrio, y si salen, salen con algún otro amigo para saber si les pasa algo por ahí por el camino" (Gilda, familiar de víctima de gatillo fácil. Conversación en marcha, 2014).

\section{Conclusiones}

Este trabajo tuvo como ejes las siguientes preguntas: ¿cómo registran subjetivamente los jóvenes cordobeses de sectores populares estas barreras materiales y simbólicas que configuran una cartografía de prohibiciones del espacio supuestamente público?, ¿qué emocionalidad producen en ellos estas experiencias de control policial cotidiano y violento?

Partimos de asumir que el espacio público es "el lugar en el que el Estado logra desmentir momentáneamente la naturaleza asimétrica de las relaciones sociales que administra y a las que sirve y escenifica el sueño imposible de un consenso equitativo en el que puede llevar a cabo su función integradora y de mediación” (DELGADO Y MALET, 2008: 61). El trazado de una topología de detenciones arbitrarias en la trama urbana local permite mostrar que cuando se hacen presentes en el centro histórico, en el shopping, en los parques de Córdoba, esos cuerpos inconvenientes por jóvenes, negros y pobres vienen a desmentir una y otra vez el proceso de naturalización e idealización por el cual se acepta como dada la existencia plena de un espacio público. Se constata así la "ficción nominal” (Delgado y MALET, 2008) de la que es objeto cuando se lo define como lugar de lo común, de encuentro sin restricciones, de acceso para todos.

En tanto se construye simbólicamente a los jóvenes de sectores populares como los únicos y directos responsables de la "inseguridad”, a través de su captura, el Gobierno de la Provincia de Córdoba tranquiliza a sectores poblacionales de clase media en el reaseguramiento de su propiedad privada. El procedimiento de "esconder la basura debajo de la alfombra", 


\section{Andrea Bonvillani}

al que refiere uno de los jóvenes, remite a la "limpieza de clase del espacio público como panacea universal de la inseguridad urbana” (WACQUANT, 2000: 12-13). La criminalización de determinados comportamientos como argumento para la exclusión de los jóvenes pobres de determinados puntos urbanos muestra evidentes resonancias con la ideología de la tolerancia cero ya aludida. La detención y exclusión del espacio urbano de los jóvenes "mal vestidos" o que cantan o tocan la guitarra o simplemente "hacen nada" procede al modo de una "modelación cultural y morfológica" (WACQUANT, 2000: 60), destinada a preservar las buenas costumbres que hacen al urbanismo y la civilidad, de acuerdo con los cánones de la cultura hegemónica. La regulación de estilos de vida y modos de sociabilidad implica inscribir una dimensión moral en el régimen de uso del espacio urbano que se conecta con la prevención de la criminalidad, porque se los considera precursores necesarios del delito y deben ser controlados y reprimidos.

Si experimentar la ciudad es un proceso de carácter corporal y sensitivo (Lindón, 2015), ¿qué sucede cuando el propio cuerpo es una marca que anticipa un límite para habitar el espacio social? Encarnar al "chivo expiatorio" (Young, 2007; CASTEL, 2013), es decir, hacer carne la atribución esencial de peligrosidad, va modelando formas particulares de habitar la ciudad, de constituirse en ciudadano, de subjetivarse desde el dolor y el miedo que producen el sentimiento de indefensión, injustica y discriminación.

A partir de las experiencias de persecución y violencia policial que se inscriben como parte de la su socialización, los jóvenes de sectores populares incorporan un régimen de uso del espacio público que consiste en la práctica naturalizada de evitar ciertas zonas de la ciudad, lo cual va aislándolos progresivamente y confinándolos en el espacio barrial o doméstico. Este cercenamiento del derecho básico a la libre circulación tiene como consecuencia una virtual privatización de los espacios que se definen como de uso común, y supone para los jóvenes un claro impedimento para disfrutar de recursos materiales y simbólicos que suelen concentrarse en el espacio céntrico de la ciudad.

La tonalidad emocional predominante con la que estos jóvenes caracterizan lo que viven cotidianamente en sus encuentros con la policía es de indefensión e impotencia, un sentimiento de "sin salida" frente a la violencia física y simbólica que padecen. Los relatos de los jóvenes muestran las inscripciones discursivas que las experiencias de persecución y hostigamiento reiterado con los agentes policiales les han dejado, las cuales dan cuenta de 
un despojamiento de su condición humana. Cuando describen estas situaciones, prevalecen formas enunciativas propias del manejo de objetos: los jóvenes pasan a ser cosas que son "paradas", "levantadas” y "llevadas” del espacio público. Estos indicios nos permiten abrir interrogantes a futuro respecto de un proceso de subjetivación juvenil fuertemente atravesado por su cosificación: jóvenes cuya existencia cotidiana está a merced de otro.

\section{Bibliografía}

ALVARADO, Arturo y SILVA, Carlos (2011). "Relaciones de autoridad y abuso policial en la ciudad de México”. [En línea]. Revista Mexicana de Sociología. 73 (3). Julio-septiembre de 2011. ISNN 0188-2503/11/07303-03. Disponible en: www.revistas.unam.mx/index.php/rms/ article/download/28724/26695

BONVILLANI, Andrea (2016) "Habitar la Marcha: notas etnográficas sobre una experiencia de protesta juvenil”. Universitas Psychologica, 14 (5).

(2015). "El Código de Faltas de la Provincia de Córdoba (Argentina) como dispositivo de poder. La construcción de la seguridad a partir de la equivalencia simbólica 'joven pobre = peligroso'”. Controversias y Concurrencias Latinoamericanas, Asociación Latinoamericana de Sociología (ALAS). 7 (11).

(2011) Travesías grupales. Algunas coordenadas para trabajar/pensar con grupos. Editorial de la Universidad Nacional de Córdoba, Córdoba.

BOITO, María y MICHELAZZO, Cecilia (2014). "Córdoba en pedazos. Habitar/circular en contextos sociosegregados”. En Estudios sociales contemporáneos, 10.

BOLATTI, Virginia, FRONTALINI REKERS, Romina, JOB, Sergio, MOYANO, Ramiro, TRUCCONE BROCCA, Magdalena, MORALES, Susana y PLAZA, Valeria (2015). "Tercer Informe de la Comisión Provincial de la Memoria. Mirar Tras los Muros. Situación de los Derechos Humanos de las personas privadas de libertad en Córdoba”. Universidad Nacional de Córdoba, Córdoba.

BROCCA, Magdalena, MORALES, Susana, PLAZA, Valeria y CRISAFULLI, Lucas (2014). "Informe Provincial 2013. Mirar Tras los Muros. Situación de los Derechos Humanos de las personas privadas de libertad en Córdoba”. Universidad Nacional de Córdoba y Universidad Nacional de Río Cuarto, Córdoba.

CALZADO, Mercedes y GALLARDO, Juan Pedro (2016). "Hacia un mapa de intervenciones electorales locales en materia de seguridad urbana (Ciudad de Buenos Aires, 2007)”. Polis, 44. CASTEL, Robert (2013) La inseguridad social ¿Qué es estar protegido? Manantial, Buenos Aires. 
CASTELLS, Manuel (1984). "La crisis, la planificación y la calidad de vida: el manejo de las nuevas relaciones históricas entre espacio y sociedad”. Revista Mexicana de Sociología, XLVI (4).

CENTRO DE ESTUDIOS LEGALES Y SOCIALES (CELS) (2016). "Hostigados: violencia y arbitrariedad policial en los barrios populares". Buenos Aires.

CHAVES, Mariana (2005). "Juventud negada y negativizada: Representaciones y formaciones discursivas vigentes en la Argentina contemporánea”. Última década, 13 (23).

DELGADO, Manuel y MALET, Daniel (2008). “El espacio público como ideología”. Ponencia presentada en el Forum español para la prevención y la seguridad urbana. Abril de 2008.

DUHAU, Emilio (2003). "División social del espacio metropolitano y movilidad residencial”. Papeles de Población. 36.

ENTEL, Alicia (2007) La ciudady los miedos. La pasión restauradora. La Crujía, Buenos Aires. JOB, Sergio y NAHAS, Estefanía (2016). "Policialización, ineficacia y discriminación políticas en seguridad en la Córdoba actual”. Rebelión. Junio de 2016. Fecha de consulta 3 de noviembre de 2016. Disponible en: http://goo.gl/0dhBG1.

LEFEBVRE, Henry (1972) El derecho a la ciudad. Península, Barcelona.

LINDON, Alicia (2015). "Del espacio público de las hexis corporales al de las afectividades brumosas y no discursivas”. Revista Latinoamericana de Estudios sobre Cuerpos, Emociones y Sociedad. 17 (7).

MARGULIS, Mario (1999). "La racialización de las relaciones de clase”. En: MARGULIS, Mario y URRESTI, Marcelo. La segregación negada. Cultura y discriminación social. Biblós, Buenos Aires.

NUÑEZ, Pedro (2006). "Patrullando la ciudad”. Política \& Sociedade, 5 (8).

PLAZA SCHAEFER, Valeria y MORALES, Susana (2013). "Seguridad y democracia: tensiones de origen. Aportes al análisis de la política de seguridad en la Provincia de Córdoba”. Estudios, 29.

REGUILLO, Rossana (2003). “Ciudadanías juveniles en América Latina”. Última Década, 19. VALLÉS, Miguel (1999) Técnicas cualitativas de investigación social. Síntesis, Madrid. WACQUANT, Loïc (2000) Las cárceles de la miseria. Manantial, Buenos Aires. YOUNG, Jock (2007) El Vértigo de la Modernidad Tardía. Un análisis del papel de la suerte en la atribución de responsabilidad penal. Didot, Buenos Aires.

ZAVALETA Alfredo, KESSLER, Gabriel, ALVARADO, Arturo y ZAVERUCHA, Jorge (2016). "Una aproximación a las relaciones entre policías y jóvenes en América Latina”. Política y gobierno. XXIII (1). 